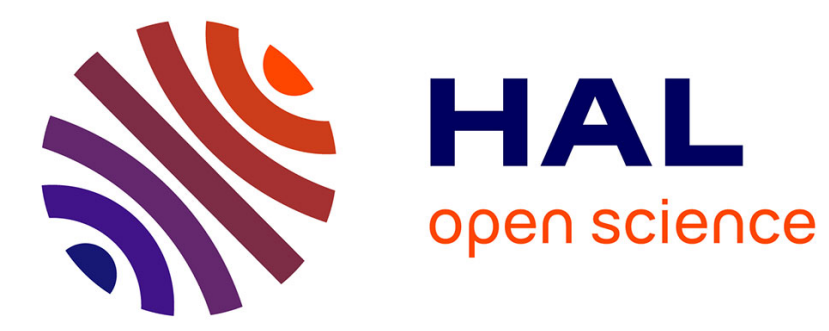

\title{
Évolution des textes, interprétations des enseignants et rapport à la discipline: le cas de l'EPS
}

\author{
Thérèse Perez-Roux
}

\section{To cite this version:}

Thérèse Perez-Roux. Évolution des textes, interprétations des enseignants et rapport à la discipline: le cas de l'EPS. Spirale - Revue de Recherches en Éducation , 2009, 43, pp.201-213. 10.3406/spira.2009.1196 . hal-01716164

\section{HAL Id: hal-01716164 \\ https://hal.science/hal-01716164}

Submitted on 27 Feb 2018

HAL is a multi-disciplinary open access archive for the deposit and dissemination of scientific research documents, whether they are published or not. The documents may come from teaching and research institutions in France or abroad, or from public or private research centers.
L'archive ouverte pluridisciplinaire HAL, est destinée au dépôt et à la diffusion de documents scientifiques de niveau recherche, publiés ou non, émanant des établissements d'enseignement et de recherche français ou étrangers, des laboratoires publics ou privés. 


\title{
Evolution des textes, interprétations des enseignants et rapport à la discipline : le cas de l'EPS
}

\author{
Perez-Roux Thérèse. Maître de conférences en Sciences de l'Education, IUFM-Université de \\ Nantes. Laboratoire du CREN.
}

\begin{abstract}
Résumé
Durant les 40 dernières années, l'EPS a vécu de profondes mutations officialisées par la parution de textes institutionnels. L'étude s'intéresse aux représentations des enseignants sur ces évolutions en lien avec les pratiques professionnelles. Une enquête par questionnaire menée dans trois académies s'est prolongée par des entretiens avec des sujets expérimentés. Au-delà des valeurs partagées par le groupe professionnel, apparaissent de nombreuses interprétations des textes et un monde relativement clivé soulignant des formes d'adhésions et de résistance. Les résultats révèlent une utilisation distanciée et modulée des aspects institutionnels et mettent en avant les marges de manœuvre des acteurs. Le rapport à la discipline s'opère entre enjeux institutionnels, interactions sociales, trajectoires individuelles et contextes professionnels plus ou moins favorables.
\end{abstract}

\section{Mots clés}

Institution, enseignant, représentations, valeurs, logiques, EPS

\section{Introduction}

\subsection{Contexte institutionnel et identité disciplinaire}

Discipline scolaire n'ayant une "spécificité que dans, par et pour l'école" (Arnaud, 1989, 29)1, l'Education Physique et Sportive (EPS) doit à la fois s'adapter et contribuer à l'évolution du système scolaire. Confrontés d'une part aux changements repérés chez les élèves dans leur rapport à l'école et d'autre part à l'hétérogénéité de ces derniers face aux savoirs, les enseignants ont à assumer plusieurs missions : transmettre connaissances et méthodes, accompagner les processus d'apprentissage, prendre en compte les différences interindividuelles pour lutter contre l'échec scolaire et contribuer à terme, à l'insertion sociale et professionnelle des élèves. L'EPS n'échappe pas à cette évolution et cherche à y répondre de façon adaptée. A ce titre, la réflexion sur les contenus et leur évaluation engagée dans les années quatre vingt dix, vise la «réussite de tous les élèves », rompant ainsi avec certains objectifs antérieurs (Instructions officielles de 1967) centrés prioritairement sur le niveau de performance des élèves, en référence à des barèmes nationaux.

De fait, durant les quarante dernières années, l'EPS a vécu de profondes mutations institutionnelles. En 1981, son entrée au ministère de l'Education Nationale a confirmé un statut de discipline d'enseignement à part entière ${ }^{2}$. Durant la période qui a suivi, sa contribution aux réflexions concernant l'évolution du système éducatif a été marquée par la parution de nombreux textes officiels (1985 et 1996 pour le collège ; 1986 et 2000 pour le lycée) $)^{3}$.

\footnotetext{
${ }^{1}$ En dehors du système scolaire, se développent des pratiques physiques compétitives ou de loisir. Celles-ci, nombreuses, sont regroupées à l'intérieur du monde de l'EPS sous le terme d'Activités Physiques et Sportives (APS). En 1996, le sigle APS intègre les activités physiques Artistiques et devient : Activités Physiques Sportives et Artistiques (APSA).

${ }^{2}$ La reconnaissance institutionnelle de la discipline est bien antérieure à cette date symbolique qui souligne le nouveau statut de discipline d'enseignement, parmi les autres et au même titre que les autres.

${ }^{3}$ Les textes renvoyant aux programmes en EPS se sont échelonnées sur plusieurs années en fonction des niveaux de classe : 1985, 1987, 1988 puis 1996, 1997, 1998 pour le collège ; 1986 puis 2000, 2001 pour les lycées. Chaque série s'inscrit, bien entendu, dans une même logique. Ces textes font toujours référence en 2008. Ils ont
} 
Perez-Roux, T. (2009). Evolution des textes, interprétations des enseignants et rapport à la discipline : le cas de l'EPS. Spirale, 43. 201-214. https://spirale-edu-revue.fr/IMG/pdf/13_perez.pdf

\subsection{D'un enseignant au rôle spécifique à un acteur intégré}

L'analyse comparative de textes officiels portant sur les missions des enseignants (1967 et 1997) ${ }^{4}$ montre deux évolutions notables ${ }^{5}$. La première concerne le passage d'une référence au sport à un ancrage sur l'élève. En 1967, on vise l'éducation aux valeurs dont les Activités Physiques et Sportives (APS) sont porteuses. Si à cette période le charisme de «l'éducateur physique » doit parvenir à faire aimer le sport - aux vertus présentées comme indéniables - il n'en va plus de même en 1997. Les élèves ont changé, les modes de pratiques sociales évoluent et les disciplines scolaires sont à construire du point de vue des savoirs fondamentaux (connaissances, méthodes) et du sens qu'elles ont pour les jeunes. Ainsi les « connaissances essentielles » (1997) dépassent la juxtaposition des APS et supposent d'entrer dans une réflexion de fond sur ce qui contribuerait à une éducation physique complète et équilibrée, dans laquelle l'élève serait amené à traverser des expériences corporelles diversifiées. Ce dernier est au coeur de la réflexion, ce qui nécessite de différencier les chemins d'apprentissage et d'engager des procédures d'évaluation formative pour le rendre acteur et le conduire vers une relative autonomie. Une pratique corporelle consciente et raisonnée remplace une pratique sportive et ses formes compétitives. L'éducation de la personne passe par des mises en jeu corporelles reliées à des données psycho-affectives, cognitives et sociales.

La deuxième évolution rend compte des attentes institutionnelles en terme de professionnalité enseignante. Spécialiste du sport et des APS avant d'entrer en formation et durant celle-ci, l'enseignant en 1967 est un sportif polyvalent qui sait et peut démontrer le «geste juste ». Respecté dans sa spécialité, il donne son avis sur le comportement des élèves lors du conseil de classe mais garde sa particularité : l'éducation du physique.

Le texte de 1997 définit de façon élargie la mission des enseignants, pour s'adapter à une complexité grandissante du système scolaire (Derouet, 1992). La société fait pression et interpelle l'école sur son efficacité. Chaque acteur en charge des élèves doit participer à leur éducation.

De fait, l'action de l'enseignant d'EPS dépasse largement le cadre de la classe et de la discipline. Il participe à une communauté éducative s'organisant autour de différents projets. Trouver sa place au sein d'une structure complexe devient un enjeu de taille. S'y investir suppose du temps et des réorganisations ; refuser d'y participer, c'est courir le risque d'être mis à l'écart.

\subsection{Continuités et changements de perspective dans les textes officiels}

L'analyse thématique des textes officiels sur la période 1967-2000 (Roux-Perez, 2001) permet de repérer des indicateurs intéressants autour desquels s'organisent les représentations professionnelles des enseignants d'EPS.

Tout d'abord l'étude conduite en 2001 met en avant une continuité des finalités et valeurs éducatives. En effet, depuis plus de quarante ans, l'EPS vise l'éducation complète de l'individu. Elle permet de «construire par la pratique, les notions de sécurité, de

été complétés en 2002 par des programmes d'enseignement de l'EPS pour les classes de CAP, BEP et Baccalauréat professionnel.

${ }^{4}$ Circulaire du 19 octobre 1967 et $\mathrm{BO} \mathrm{n}^{\circ}$ 22, 29 mai 1997 : analyse comparative de ces textes a été réalisée par T.Roux-Perez (2001, p.608).

${ }^{5}$ Il n'est pas question ici de développer une analyse historique précise des textes officiels, déjà réalisée par des auteurs comme J-Y Nerin (2003) car au programme des concours de recrutement d'enseignants d'EPS. Notons simplement de grandes tendances supposant un infléchissement des pratiques professionnelles et de fait, des interprétations multiples de la part des acteurs censés mettre en œuvre les recommandations officielles. 
Perez-Roux, T. (2009). Evolution des textes, interprétations des enseignants et rapport à la discipline : le cas de l'EPS. Spirale, 43. 201-214. https://spirale-edu-revue.fr/IMG/pdf/13_perez.pdf

responsabilité, de coopération. En offrant des occasions concrètes d'accéder aux valeurs morales et sociales, elle participe à l'éducation à la citoyenneté ». Ces finalités traversent les différents textes régissant la discipline. Par ailleurs, les objectifs de l'EPS, organisés autour de trois pôles, s'inscrivent eux aussi dans une continuité : développement de la motricité, appui sur une culture et acquisition de la santé donnent à la discipline une pertinence scolaire.

Au-delà de ces stabilités faisant consensus, la réflexion sur les processus d'apprentissage a participé d'un mouvement de rupture plus radical. En 1967, l'élève n'est évoqué qu'à travers sa motivation quasi automatique à pratiquer le sport sous des formes compétitives. Contrôle des résultats pour repérer les progrès, tests des performances organisent l'année. Dès 1985, une bascule s'opère. L'élève n'est plus pensé sous un modèle unique mais dans sa diversité. Des critères de réussite et des critères de réalisation simples l'amènent à suivre l'évolution de ses apprentissages et à construire de nouvelles habiletés motrices. L'enseignant propose des buts clairement identifiés, différenciés, accessibles à chacun et auto-évaluables. Ainsi, l'objet de l'enseignement n'est plus les APS pour elles-mêmes mais l'élève, dont les ressources sont prises en compte pour lui permettre de réussir. La pédagogie du contrat est valorisée. Les textes de 1996 confirment cette tendance, en demandant un engagement plus réfléchi dans les pratiques corporelles en fin de collège.

Cette prise en compte radicale d'un élève acteur de ses apprentissages et d'un enseignant cherchant à donner du sens à ses interventions conduit à des changements au sein de la discipline. Plusieurs indicateurs ont été retenus pour repérer les évolutions entre 1967 et 2000: constitution d'un programme ; élaboration d'un projet pédagogique ; changement du statut accordé aux APSA ; structuration des contenus d'enseignement ; clarification des modalités d'évaluation ; gestion des groupes et de la mixité. Trois de ces indicateurs sont présentés cidessous de manière à expliciter l'esprit des transformations induites par les textes.

Tout d'abord, la nécessité de construire un projet pédagogique d'Education Physique et Sportive apparaît progressivement. En 1967, le professeur élabore son enseignement à partir d'une simple programmation des APS. Un nombre d'heures est donné, à titre approximatif, pour les différentes activités et en fonction du niveau de cursus. Le programme d'établissement doit tenir compte des «objectifs proprement scolaires tels que la préparation aux épreuves sportives ». Il s'articule, par ailleurs avec les calendriers sportifs. C'est donc le sport qui organise prioritairement l'enseignement. En 1985, le projet s'impose dans les textes et doit se construire en équipe pour rendre l'enseignement plus cohérent et plus lisible pour l'ensemble des acteurs. Il dépasse la simple programmation des activités physiques et s'intéresse aux contenus de formation et à leurs modes d'évaluation explicités en vue du contrôle en cours de formation. En 1996, il se fonde à la fois «sur les acquisitions antérieures des élèves et sur leurs besoins» et "sur les objectifs généraux de l'établissement». Si le programme, organisé autour de trois types de compétences ${ }^{6}$ constitue, à cet égard, un ensemble de références pour l'équipe d'EPS, il n'en demeure pas moins que le collectif des enseignants doit se mettre d'accord sur une orientation claire et des modalités de mise en œuvre pertinentes.

Cette réflexion sur le projet pédagogique suppose de redéfinir le statut des Activités Physiques et Sportives. En effet, si «le sport tient la plus grande place » en 1967 et si la pratique des APS tend à devenir la finalité première de l'EPS, le texte de 1985 précise que "l'EPS ne se confond pas avec les APS qu'elle propose et organise ». Le choix des activités s'effectue par le jeu combiné de leurs caractéristiques, et des types de relations qu'elles engagent entre le sujet et l'environnement physique et humain. En 1996, la nature des activités

\footnotetext{
${ }^{6}$ Il s'agit de compétences spécifiques à une activité, propres à un groupe d'activités répondant à la même logique comme par exemple les sports collectifs et générales renvoyant davantage aux finalités éducatives.
} 
va supposer chez l'élève différents types d'adaptation permettant à chacun de s'éprouver et de vivre des expériences variées et originales.

Enfin, la notion d'évaluation s'affine et se complexifie. L'évaluation formative n'apparaît pas dans les textes de 1967 où la compétition reste le meilleur moyen de contrôler les résultats obtenus. On repère un déplacement vers les processus d'acquisition de l'élève dès 1985 . En effet, ce dernier «apprend à évaluer les difficultés rencontrées et la pertinence des effets de ses actions». Les textes de 1996 renforcent cet aspect, valorisant «un engagement plus réfléchi de l'élève dans ses apprentissages ».

L'évaluation sommative, toujours présente, va faire l'objet en vingt ans, d'une réelle mutation. En 1967, il est question de «compositions » où l'objectif reste la préparation des épreuves sportives. Athlétisme, gymnastique, natation sont évaluées avec des tables de mesure nationales. Il y a donc centration sur la motricité performante.

Une rupture radicale s'opère en 1985 lorsque la motricité ne constitue plus que deux tiers de la note du brevet des collèges ; elle intègre la performance avec des barèmes propres à l'établissement; d'autre part elle s'intéresse aux conduites motrices (niveaux d'habileté, niveaux de compétences) qui structurent l'apprentissage. Par ailleurs, les connaissances dans l'action et l'investissement pour progresser sont évaluées pour un tiers de la note. Cette ouverture de la note permet d'encourager les élèves et, aux yeux des législateurs, de rendre la discipline plus juste, car moins centrée sur les seules aptitudes physiques des élèves. Ce mode d'évaluation reste inchangé dans les textes de 1996. Il permet aux enseignants de valoriser l'effort, l'engagement dans l'action, les progrès et, par ce fait, de motiver davantage des élèves qui n'étaient pas très performants ${ }^{7}$.

L'étude s'intéresse à la manière dont les évolutions repérées dans les textes officiels sont interprétées par les enseignants d'EPS et « réinvesties » dans les pratiques. Les différents points évoqués ci-dessus constituent donc des «objets professionnels » (Moliner, 1993) considérés comme des indicateurs significatifs pour appréhender les représentations des enseignants.

\section{Repères théoriques et méthodologiques}

\subsection{Des représentations professionnelles entre dynamique institutionnelle et réalités des pratiques}

Les enseignants d'EPS véhiculent un ensemble de valeurs et de croyances, partagées avec d'autres enseignants, compatibles avec leurs pratiques professionnelles ou personnelles et repérables à travers leurs représentations du métier, des autres et d'eux-mêmes. En construisant ces représentations, ils réduisent d'éventuelles dissonances avec leur environnement humain et institutionnel. Les théories de la consistance cognitive (Festinger, 1957) en lien avec celles des représentations sociales et professionnelles éclairent ce point de vue (Moscovici, 1961 ; Jodelet, 1989 ; Abric, 1994 ; Blin, 1997 ); elles permettent d'étudier la manière dont les enseignants perçoivent l'évolution de leur discipline et justifient leurs positions vis-à-vis du métier. Porteuses de valeurs, ces représentations donnent sens à la pratique et légitiment une certaine «vision du monde»; elles servent à (ré)agir face à l'environnement tout en conservant un équilibre cognitif, dans un contexte professionnel particulier.

\footnotetext{
${ }^{7}$ Cet aspect a pu être ensuite considéré comme une dérive par l'institution qui ne souhaitait sans doute pas que l'évaluation des connaissances et de l'investissement devienne un moyen de compensation et d'ajustement des notes, supprimant des écarts trop importants entre les élèves en terme de motricité.
} 
Ainsi, les représentations professionnelles peuvent être considérées comme des grilles de lecture de cet environnement spécifique. Définies comme «un ensemble de cognitions descriptives, prescriptives et évaluatives portant sur des objets significatifs, utiles à l'activité professionnelle et organisées en un champ structuré présentant une signification globale » (Blin, 1997, 89), les représentations professionnelles sont en lien avec la pratique, l'identité et les savoirs professionnels, avec lesquels elles forment les composantes essentielles du système professionnel (Mias, 1998).

Cette approche nous amène à questionner le rapport à la discipline - et plus largement au métier - perçu à travers des représentations, envisagées comme des systèmes de relation, d'interprétation et ancrées dans un réseau de significations (Charlot, 1997) ${ }^{8}$.

Plus largement, chaque individu peut être considéré comme un acteur capable de maîtriser consciemment, au moins en partie, son rapport au monde. Pour Dubet (1994), chaque expérience sociale résulte de l'articulation de trois logiques de l'action qui lient l'acteur à chacune des dimensions d'un système : dans la «logique de l'intégration », l'acteur se définit par ses appartenances, vise à les maintenir ou à les renforcer pour s'intégrer à un collectif. Dans la «logique de la stratégie », l'acteur s'organise pour réaliser au mieux ses intérêts dans un espace collectif conçu comme un marché potentiel. Dans la «logique de la subjectivation », l'acteur se définit avant tout comme un sujet critique, mettant à distance ses appartenances collectives.

La contribution s'intéresse donc aux transactions entre logique institutionnelle et logique des acteurs. Pour gérer d'éventuelles dissonances entre ces deux logiques, les enseignants mobilisent nombre de représentations sur l'exercice du métier. Se positionnant de façon contrastée face aux changements institutionnels, ces derniers mettent en œuvre des formes de pratiques pédagogiques qui semblent se modifier proportionnellement au degré d'adhésion éprouvé vis-à-vis des textes.

\subsection{Procédure utilisée}

Au niveau méthodologique, une enquête par questionnaire a été proposée en 1998 dans trois académies $^{9}(n=381)$. Dans ce document, une série de questions ouvertes avait pour but de comprendre comment les enseignants d'EPS analysaient l'évolution de la discipline en le référant : a) au degré de motivation exprimé pour l'enseignement; b) à l'interprétation des textes officiels ; c) aux changements observés dans les pratiques professionnelles depuis l'entrée dans le métier.

Dans le prolongement de ce travail (2000), nous avons réalisé 21 entretiens semi-directifs avec des enseignants expérimentés et formés sur la base de textes antérieurs ${ }^{10}$, pour mettre à l'épreuve un certain nombre de catégories construites à partir des modes d'implication professionnelle privilégiés par les enseignants (Roux-Perez, 2003). Dans ce corpus des 21 entretiens initialement réalisés, douze ont été retenus selon des critères de représentativité à l'échelle nationale (sexe, type d'établissement, niveau de diplôme). Nous avons opté pour les enseignants les plus caractéristiques au regard des grandes tendances au sein de la profession, à l'appui de la typologie construite précédemment. Une analyse structurale du discours inspirée des travaux de Demazière et Dubar (1997) a été conduite dans le but d'appréhender plus finement les représentations et valeurs sous-jacentes fondant un rapport singulier à la

\footnotetext{
${ }^{8}$ Pour B. Charlot (1997, p. 93) « le rapport au savoir est rapport au monde, à l'autre et à soi-même d'un sujet confronté à la nécessité d'apprendre ». Il semble intéressant de transposer cette définition dans le cadre du rapport au métier des enseignants.

${ }^{9}$ Le choix s'est porté sur les académies de Corse, de Créteil et de Nantes, diversement engagées, à l'époque, dans la «dynamique institutionnelle » et la réflexion sur les textes à venir.

${ }^{10}$ La majorité des enseignants était âgée au moment de l'enquête de 40 à 50 ans.
} 
discipline. Plusieurs indicateurs repérés dans les textes officiels et suscitant nombre de débats dans le groupe professionnel étudié, ont structuré notre investigation et ont constitué autant d'objets professionnels permettant de mettre en lumière, chez les enseignants interrogés, les représentations de la discipline, le type de rapport aux textes et leur possible traduction dans les pratiques.

\section{Résultats}

Les résultats soulignent la complexité des relations des enseignants d'EPS avec l'évolution disciplinaire, elle-même traduite en partie dans les textes officiels.

\subsection{Représentations professionnelles et valeurs partagées par le groupe professionnel}

Les résultats issus du questionnaire mettent en relief une relative cohérence en lien avec l'évolution repérée dans les textes. Pour les enseignants d'EPS, les changements disciplinaires sont essentiellement liés à la "réflexion sur la didactique de l'EPS" (28\%), à la "centration sur l'élève" (22\%) et à "l'évolution de l'évaluation" (21\%). Les relations enseignant-élèves se sont modifiées et l'EPS, discipline scolaire ne se confond plus avec le sport. En se diversifiant, les pratiques (APSA) constituent des sources de motivation supplémentaires pour les élèves. Enfin, un travail d'équipe leur semble incontournable pour construire le projet pédagogique d'EPS. La logique institutionnelle, très présente, semble donc servir de référence mais reste toujours ouverte aux interprétations. C'est donc avec ou contre cette logique que les enseignants d'EPS peuvent se positionner pour défendre leur conception de la discipline.

Ainsi, une permanence fondatrice de l'identité professionnelle collective traverse toutes les réponses aux questions ouvertes ; elle est organisée autour des valeurs éducatives inscrivant la mission de l'enseignant d'EPS dans une logique de formation, centrée sur une relation enseignant-élève privilégiée dans cette discipline. Enfin, ils entrent dans une demande d'éducation qui leur est aisément dévolue, y compris par les autres enseignants davantage préoccupés par les savoirs disciplinaires (Dorville, 1991). Cet ensemble d'éléments semble constituer une sorte de « label » ${ }^{11}$ légitimant l'intégration de l'EPS au sein du système scolaire.

\subsection{Rapport à l'institution : entre adhésion et résistances}

Au-delà des représentations partagées structurant le groupe professionnel, l'étude montre des variations importantes dans l'interprétation des textes officiels. Sept des douze enseignants suivis dans l'étude qualitative évoquent les textes officiels comme un réel point d'appui sans occulter certaines difficultés à les mettre en œuvre. Soucieux de penser l'enseignement à partir des ressources des élèves, ces enseignants s'engagent dans une réflexion, le plus souvent collective, sur la cohérence des contenus et les modalités d'évaluation qui seront choisies. Parmi eux, certains, plus nuancés, considèrent les textes comme des objets « intéressants mais relativement vagues et imprécis », sorte de "grosses balises, laissant une marge de manœuvre importante »; ils les lisent, les interprètent et disent s'en inspirer plus ou moins dans la réalité de leurs pratiques. Quoi qu'il en soit, ces enseignants conservent une forme de vigilance quant à l'articulation textes-terrain, moins évidente que ne le laisseraient

\footnotetext{
${ }^{11}$ Le terme «label» est employé au sens de «marque qui garantit l'origine ou la qualité d'un produit » (dictionnaire Petit Robert) ; transposé dans notre étude, le «produit » concerne l'EPS et ce qui pourrait fonder sa légitimité institutionnelle.
} 
Perez-Roux, T. (2009). Evolution des textes, interprétations des enseignants et rapport à la discipline : le cas de l'EPS. Spirale, 43. 201-214. https://spirale-edu-revue.fr/IMG/pdf/13_perez.pdf

entendre les discours des corps d'inspection : «l'inspecteur a mis la profession en mouvement, c'est sur, mais il faut aussi discuter avec lui de la réalité de tous les jours ».

Les autres enseignants interrogés affichent une réelle distance vis-à-vis des textes. Lus partiellement ou en surface, ils ne servent qu'à entrer dans l'exigible : le projet pédagogique et les modalités d'évaluation sont éventuellement formalisés - notamment au Lycée - pour répondre aux attentes institutionnelles mais restent peu opérants dans les pratiques. Enfin, pour un petit nombre d'enseignants, qui se disent «en résistance » avec l'évolution disciplinaire, les textes ne sont pas lus car situés au niveau des discours et très éloignés des pratiques: «nous, c'est pas la tête dans les nuages, c'est plutôt les pieds dans la boue!». Dans ce cas l'inspecteur, représentant de l'institution, devient l'objet de toutes les focalisations alors que l'on sait son faible poids dans les pratiques quotidiennes. Il est perçu comme l'ultime miroir invitant à lire les décalages avec les recommandations officielles. Pour peu que ce dernier insiste et s'oppose à une éducation physique qui ne serait qu'une juxtaposition d'activités, il met momentanément le professeur en difficulté. Les discours sont alors extrêmement tranchés à son encontre : «il a cassé l'ambiance d'avant », "il a complètement nié l'expérience des anciens ». Plusieurs enseignants de ce groupe déplorent une faible prise en compte, par l'inspecteur, des caractéristiques «réelles » de leurs contextes de travail et vivent la situation d'inspection comme une intrusion dans un quotidien professionnel ressenti comme difficile. En conséquence, la grille d'évaluation, jugée formelle et inopérante, est remise en cause de façon catégorique.

\subsection{Interprétation des textes et logique des acteurs}

Au-delà de ces deux tendances, existent bien évidemment des variations individuelles car les enseignants appréhendent aussi les textes en fonction de leur propre histoire face aux savoirs scolaires et aux pratiques sportives (Roux-Perez, 2006). Les discours de trois enseignants d'une même génération (formés sur la base des instructions officielles de 1967) rendent compte de ce rapport différencié à la logique institutionnelle et à ses éventuelles incidences dans la pratique.

Colette, 49 ans, enseigne en ZEP. Très impliquée au niveau de l'établissement comme au niveau syndical, elle se dit plutôt en phase avec les textes en EPS, bien qu'elle perçoive un alourdissement progressif des tâches à assumer dans l'établissement, au détriment d'un travail de fond dans sa discipline. Très sensible à la continuité et à la cohérence disciplinaire, elle œuvre pour l'élaboration du projet pédagogique et regrette certaines résistances chez ses collègues. Son souci d'accompagner des élèves d'un collège difficile l'amène à valoriser l'évaluation formative. Elle y retrouve des enjeux de formation communs à d'autres disciplines et cette cohérence lui semble nécessaire face à «des élèves auxquels il faut donner des repères ». Enfin, la réflexion sur l'adaptation des contenus et sur les critères d'évaluation reste au cœur de ses préoccupations pédagogiques. Si Colette se «casse la tête pour que les élèves participent, comprennent bien ce qui est attendu... alors que des fois ils n'en ont rien à faire », c'est parce qu'elle croit, fondamentalement, que l'élève doit "trouver du sens à ce qu'il fait, à la manière dont il est évalué ».

François, 51 ans, enseigne en Lycée. Il énonce clairement sa distance aux textes qui «sont partis dans tous les sens... on t'impose des façons d'évaluer mais on te donne pas le truc». Sensible au contrôle continu qui l'aide à enrôler les élèves dans le travail et l'effort, cet enseignant bute sur une réflexion concernant les contenus à évaluer avec des modalités explicitées, pertinentes et adaptées. En ce sens, il privilégie «une évaluation globale » contre une évaluation «critériée » qu'il qualifie de formaliste : "quand je vois calculer le coefficient de dispersion des volants sur un terrain, je trouve ça navrant!». Cette remarque montre un faible intérêt à propos des procédures utilisées pour faire comprendre aux élèves le type de jeu 
qu'ils utilisent en badminton et pour les aider à construire des stratégies d'attaque ou de défense. Par ailleurs, pour cet enseignant, la mixité des groupes classe pose problème : "quand j'ai une classe mixte, j'ai l'impression d'un nivellement par le bas ». On perçoit à travers l'expression de ce problème une difficulté à différencier les propositions, renforcée par un choix d'activités dans lesquelles François se sent compétent : athlétisme, natation, sports collectifs et sports de raquettes. Les autres activités, qu'il refuse d'enseigner, lui semblent au final «très éloignées des centres d'intérêt des élèves ».

Michèle 49 ans, enseigne dans un collège rural. Préalablement institutrice, elle envisage les textes comme un réel point d'appui. Si le projet pédagogique constitue un moyen de mise en cohérence de l'enseignement, la réflexion sur les contenus et leur évaluation reste un élément fondamental. Des difficultés de mise en œuvre sont évoquées : élèves de collège «influencés par une culture sportive très médiatisée », problème des apprentissages techniques incontournables et décontextualisés. L'importance que les élèves semblent accorder à la note vient perturber la logique qu'elle défend, centrée sur le développement de l'enfant. Cette démarche revendiquée s'inscrit en continuité avec ce qu'elle mettait en œuvre à l'école primaire et dont elle regrette «le côté ludique et ouvert».

\section{Discussion}

\subsection{Derrière l'unité apparente, une variété de points de vue}

Le rapport des enseignants d'EPS à une discipline fortement structurée par les textes officiels est perçu à travers des représentations professionnelles; en lien avec les pratiques, ces représentations s'organisent dans un réseau de significations que les entretiens permettent d'appréhender.

Ainsi, les valeurs éducatives et la relation à l'élève semblent révéler une forme de consensus chez les enseignants d'EPS et renvoient à une logique de l'intégration (Dubet, 1994). L'appui sur les finalités constitue par ailleurs un levier important pour la profession et sa reconnaissance. En effet, l'EPS, pour ne pas se confondre avec les pratiques sportives ou de loisir extérieures au monde scolaire, souvent plus attrayantes pour ceux qui les choisissent, doit insister sur les enjeux éducatifs de la discipline et la prise en compte des potentialités réelles de l'élève.

Mais, dans le même temps, cette position oblige les enseignants à mettre à distance une spécificité motrice qui les a construits et qui n'est ni valorisée ni valorisante dans le contexte scolaire. Lorsque la référence au corps, relativement occultée dans les réponses au questionnaire $^{12}$, est abordée à l'occasion des entretiens, les enseignants expriment des positions différentes. En effet, si ces derniers se réfèrent souvent aux finalités, ils empruntent des chemins radicalement différents pour y parvenir, sélectionnent ce qui fait sens pour réduire les dissonances, ou dissocient les discours de la pratique au quotidien.

On remarque par exemple que les APSA relèvent bien d'une culture commune. Leur pratique au sein des clubs et dans le cursus de formation fédère les enseignants et participe d'un fonds commun partagé (Couturier et Duret, 2000). Mais le statut qui leur est donné dans le cadre de l'enseignement est ensuite très variable d'un enseignant à l'autre. L'enquête permet de repérer des enseignants qui revendiquent une orientation sportive. Donnant aux APSA un statut particulièrement important, ils proposent à leurs élèves des progressions proches des logiques

\footnotetext{
${ }^{12}$ Dans l'enquête par questionnaire, la variable 52 permettait de caractériser l'EPS. Ce qui renvoie à la spécificité motrice n'apparaît qu'en troisième et quatrième position : 1) valeurs éducatives : santé, sécurité, citoyenneté, autonomie, citoyenneté $(16 \%)$; 2) logique institutionnelle: programme, projet pédagogique, didactique, évaluation $(12 \%) ; 3)$ développement moteur $(10 \%) ; 4)$ APSA $(7 \%)$.
} 
Perez-Roux, T. (2009). Evolution des textes, interprétations des enseignants et rapport à la discipline : le cas de l'EPS. Spirale, 43. 201-214. https://spirale-edu-revue.fr/IMG/pdf/13_perez.pdf

fédérales. D'autres enseignants ont tendance à s'intéresser davantage aux acquisitions de l'élève, l'APSA ne devenant qu'un moyen de se développer, de progresser avec les autres et vis-à-vis de soi-même. Cela suppose un traitement didactique particulier où l'enseignant n'hésite pas à transformer l'activité pour lui donner une «forme scolaire », proche des possibilités effectives des élèves (modification des espaces et du temps, du nombre de joueurs, du règlement, etc.). Enfin, apparaissent des enseignants qui s'inscrivent avant tout dans une approche polyvalente, jugée plus motivante, permettant à l'élève de construire des savoir-faire diversifiés dans des APSA porteuses d'enjeux de formation différents. Le but est alors de faire une éducation plurielle et équilibrée en EPS en prenant appui sur des activités complémentaires.

Cette mise en relief des différences en terme de rapport à la logique institutionnelle, repérée au prisme des choix méthodologiques du chercheur, n'empêche nullement les enseignants d'EPS, en tant que groupe professionnel, d'afficher une relative cohérence pour l'extérieur. Lorsque la discipline est en danger (diminution des horaires, perte de certains acquis comme l'animation de l'association sportive, etc.) ils sont capables de se mobiliser. Pour dépasser les clivages et retrouver une unité, ils se réfèrent aux finalités de la discipline, aux valeurs éducatives et à la relation privilégiée avec les élèves, se situant alors comme des acteurs particuliers et indispensables au sein de l'école.

\subsection{Tensions au sein du groupe professionnel et spécificités revendiquées}

$\mathrm{Au}$ fil des entretiens, des tensions émergent, révélant des réseaux de représentations constitutifs de logiques de pensée et d'action (Roux-Perez, 2004).

Certains enseignants se disent globalement en accord avec l'évolution disciplinaire. La prise en compte des élèves à leur niveau de ressources motrices suppose de réfléchir à un traitement didactique des APSA et à des formes d'évaluation adaptées. Le projet pédagogique est envisagé comme une mise en cohérence permettant progressivité et continuité du curriculum, y compris lorsqu'un remplaçant prend le relais de l'enseignant : enseigner est une profession qui suppose conceptions et mises en œuvre, analyses et régulations. Le «terrain » est alors évoqué comme « résistant » parce que les élèves contournent parfois le projet de l'enseignant et n'entrent pas dans les processus d'apprentissage attendus. Mais des ajustements, souvent construits avec l'aide du collectif en fonction des problèmes repérés, amènent les enseignants à adapter leur pratique et à garder l'essentiel de ce à quoi ils tiennent en terme de contenus.

D'autres enseignants se situent en décalage avec l'évolution disciplinaire et opèrent un repli sur la spécificité motrice de la discipline. Nombre d'entre eux, reconnus pour leurs compétences sportives, ont suivi une formation initiale relativement courte dans laquelle pratique et pédagogie sportives étaient valorisées (Perez-Roux, 2007). Ces enseignants, souvent plus âgés, sont critiques vis-à-vis de la formation continue actuelle qu'ils estiment trop didactique et s'en désintéressent. Valorisant la pratique, le jeu, la technique, ils défendent l'idée que (tous) les élèves apprécient les pratiques sportives. Les APSA doivent être maîtrisées pour être enseignées, au risque d'en restreindre le choix. Le projet pédagogique est appréhendé comme un cadre formel qui n'infléchit en rien les options individuelles. Enseigner est avant tout un art qui se vit dans l'interaction avec les élèves.

Dans cette logique, la gestion des groupes mixtes pose problème, notamment lorsque sont programmées des APSA plus traditionnelles et performatives (de type athlétisme, natation ou sports collectifs). La prise en compte du seul résultat montre alors des différences entre garçons et filles, vécues comme irréductibles. Elle se double souvent d'un oubli de certaines activités moins «classantes» parce que moins «masculines»: activités de pleine nature, activités physiques artistiques ou activités gymniques. 
Perez-Roux, T. (2009). Evolution des textes, interprétations des enseignants et rapport à la discipline : le cas de l'EPS. Spirale, 43. 201-214. https://spirale-edu-revue.fr/IMG/pdf/13_perez.pdf

De la même manière, l'évaluation formative est perçue comme une perte de temps par les enseignants qui valorisent les pratiques pour elles-mêmes et ne voient pas l'utilité pour l'élève de se situer, de réfléchir sur son action. Dans ce cas, les procédures d'observation, de coévaluation sont considérées comme inefficaces. De plus, elles nécessitent la clarification de critères pour passer de l'implicite à l'explicite. Une telle démarche pose problème à ces enseignants ou reste, pour eux, sans fondement.

Cette approche nous renvoie à la «logique de la stratégie» (Dubet, 1994) qui amène les individus à interpréter la situation, à gérer les ressources du système, à se mobiliser en fonction des opportunités qui leur sont offertes, tout en s'assurant du maintien des règles du jeu pour une intégration minimale. Objectifs, intérêts et reconnaissance leur permettent alors d'investir des espaces de pouvoir et de s'y réaliser. Lorsque les investissements choisis positionnent les enseignants trop loin de la logique institutionnelle, les valeurs partagées (rôle d'éducateur, relation à l'élève) «deviennent des ressources permettant de dissimuler la réalité plus profonde des passions et des intérêts authentiques mais inavouables » (Dubet, 1994, $125)$.

\subsection{Relation modulée aux textes et stratégies des acteurs}

Cette enquête éclaire la manière dont l'enseignant investit son métier et se saisit des transformations impulsées par les textes officiels en fonction des espaces/contextes qui lui ont permis de se construire.

En fait, les textes sont suffisamment consensuels et ouverts pour permettre une circulation relativement souple et rendre possibles des marges d'interprétation. Tel ou tel aspect va faire sens pour l'enseignant et l'amener à infléchir ses pratiques, de façon plus ou moins approfondie. Si la formation continue peut jouer un rôle important d'accompagnement, le contexte professionnel s'avère aussi plus ou moins porteur : enseignement en collège ou en lycée, conditions de travail, dynamique de l'équipe, place et reconnaissance de l'EPS dans l'établissement, etc. autant d'aspects qui jouent en faveur ou en défaveur d'une relation « constructive » aux textes officiels.

Au final, les enseignants justifient et rationalisent leurs choix au travers de représentations sur le métier, sur les élèves et sur l'évolution de l'EPS. Cette organisation du monde s'effectue en lien avec leur propre histoire sportive, scolaire, professionnelle et intègre les interactions qu'ils ont nouées avec les autres, accompagnées d'affects positifs ou négatifs. Ce positionnement est porteur de valeurs construites au fil du temps qui mobilisent le sujet et donnent sens à son action. Inscrit dans une « logique de la subjectivation » (Dubet, 1994), ce dernier fait des choix pour conserver le sentiment d'être en cohérence avec lui-même, mettant à distance, s'il le faut, le rôle que l'institution voudrait lui voir jouer et dont il interprète le contenu à sa mesure.

\section{Conclusion}

Le rapport des enseignants aux textes est relativement complexe et se joue à différents niveaux : institutionnel, collectif et individuel. Les enseignants se saisissent des éléments structurants pour développer ensuite, en fonction des contextes de travail et de leurs propres références, les registres qui les intéressent.

Au-delà de ce constat, le risque d'une réflexion sur la relation aux textes officiels serait de réifier l'évolution d'une discipline qui n'existe pas en soi, indépendamment des acteurs qui la font (les législateurs, les inspecteurs mais aussi les formateurs, les enseignants d'EPS) et de ceux qui la regardent ou la vivent (les autres enseignants, les personnels de direction, les élèves) ; ces différents acteurs surgissent constamment dans le discours des sujets interviewés. Les enseignants, au centre de cette profession contribuent eux-mêmes à faire évoluer la 
Perez-Roux, T. (2009). Evolution des textes, interprétations des enseignants et rapport à la discipline : le cas de l'EPS. Spirale, 43. 201-214. https://spirale-edu-revue.fr/IMG/pdf/13_perez.pdf

discipline, leur action s'inscrivant dans plusieurs logiques simultanément. Ayant incorporé normes et valeurs (y compris dans leurs contradictions), ils les incarnent par leur action. En particulier, lorsqu'ils parlent de l'évolution disciplinaire, ils le font souvent en projetant les dimensions significatives de cette évolution dans tel groupe de collègues, tel formateur, tel inspecteur. La discipline relève à la fois de ce qui est abstrait, idéalisé, et de ce qui est concret, vécu ; elle s'appréhende dans une sorte de synthèse du dedans et du dehors, entremêlant enjeux institutionnels, interactions sociales, trajectoires individuelles et contextes professionnels plus ou moins dynamisants (Perez-Roux, 2006).

\section{Bibliographie}

Abric, J.C. (1994). Pratiques sociales et représentations. Paris, PUF.

Arnaud, P. (1989). «Contribution à une histoire des disciplines d'enseignement »-Revue

Française de Pédagogie, 89 (29-34).

Blin, J.F. (1997). Représentations, pratiques et identité professionnelle. Paris, L'harmattan. Charlot.B (1997). Du rapport au savoir. Paris, Anthropos.

Couturier, C. \& Duret, P. (2000). Enseigner l'EPS : identité professionnelle à l'épreuve du sport. Paris, Centre National d'Etudes EPS et Société.

Demazière, D \& Dubar, C. (1997). Analyser les entretiens biographiques. Paris, Nathan.

Derouet, J-L. (1992). Ecole et justice. De l'égalité des chances aux compromis locaux. Paris, Métailié

Dorvillé, C. (1991). «Représentations de l'enseignant d'EPS par les parents d'élèves et les Professeurs »-Revue STAPS, 24 (7-23).

Dubet, F. (1994). Sociologie de l'expérience. Paris, Seuil.

Jodelet, D. (1989). Les représentations sociales. Paris, PUF.

Mias, C. (1998). L'implication professionnelle dans le travail social. Paris, L'Harmattan. Moliner, P. (1993). «L'induction par scénario ambigu. Une méthode pour l'étude des représentations sociales »-Revue internationale de psychologie sociale, 6 (7-21).

Moscovici, S. (1961). La psychanalyse, son image et son public. Paris, PUF.

Nérin, J-Y. (2003). « Les Instructions officielles et l'EPS au XXe siècle »-Dossier EPS, 44.

Perez-Roux, T. (2006). "Identité professionnelle des enseignants : entre singularité des parcours et modes d'ajustement aux changements institutionnels »-Revue Savoirs, 11 (107123).

Perez-Roux, T. (2007). «Tenir comme enseignant d'EPS : entre modèles professionnels et implication personnelle »- in A. Gonin-Bolo (sdr). Parcours professionnels : des métiers pour autrui entre contraintes et plaisir (53-74). Paris, Belin.

Roux-Perez, T. (2001). Des processus de construction de l'identité professionnelle des enseignants d'Education Physique et Sportive : entre appartenance au groupe, expériences professionnelles singulières et recompositions identitaires à l'échelle du temps. Thèse de $3^{\circ}$ cycle en Sciences de l'Education, Université de Nantes, non publiée.

Roux-Perez, T. (2003). «Identité professionnelle et modes d'implication privilégiés chez les enseignants d'EPS »-Revue Les Sciences de l'Education. Pour l'Ere Nouvelle, XXXVI, 4 (37-68).

Roux-Perez, T. (2004). «L'identité professionnelle des enseignants d'Education Physique et Sportive: entre valeurs partagées et interprétations singulières ». Revue STAPS, 63 (75-88).

Roux-Perez, T.( 2006). «Processus identitaires dans la carrière des enseignants : deux études de cas en EPS »-Revue STAPS, 72 (35-47). 